\title{
All-optical gates facilitated by soliton interactions in a multilayered Kerr medium
}

\author{
Jacob Scheuer \\ Department of Electrical Engineering, Technion, Haifa 32000, Israel, and Department of Applied Physics \\ MC 128-95, California Institute of Technology, Pasadena, California 91125 \\ Meir Orenstein \\ Department of Electrical Engineering, Technion, Haifa 32000, Israel
}

\begin{abstract}
Received February 19, 2004; revised manuscript received December 23, 2004; accepted January 10, 2005
All-optical soliton logic operations, facilitated by incoherent interactions of multiple spatial solitons with nonlinear interfaces, are proposed and analyzed. A particlelike model, validated by beam propagation simulations, was developed for calculating the soliton trajectories and was employed for the analysis of the soliton-based logic gates. (C) 2005 Optical Society of America

OCIS codes: $190.3270,190.4350,190.4420,190.5530$.
\end{abstract}

\section{INTRODUCTION}

During the past few decades much attention and effort has been focused on the possibility of all-optical processing and computing by using optical soliton-based switches and logic gates. Because of its particlelike characteristics, a soliton (either spatial or temporal) is a natural candidate for the implementation of an optical bit. Most soliton-based logic gates and switches employ direct interactions between solitons, which alter the phase profiles of the interacting soliton through cross-phase modulation. ${ }^{1}$ This phase shift corresponds to a modification of the frequency for a temporal soliton or to a modification of the propagation direction for a spatial soliton.

Various methods that exploit the modification of the solitons' trajectories by this interaction for switching and all-optical logic were proposed and demonstrated. ${ }^{1-14}$ Soliton-dragging gates, ${ }^{15-24}$ for example, are based on the interaction of orthogonally polarized solitons propagating in a birefringent medium. Soliton trapping phenomena were used to demonstrate all-optical switching and logic operations. ${ }^{24,25}$ Other concepts, based on interactions in saturable nonlinear media ${ }^{14,26}$ wave mixing, ${ }^{15,16}$ and Manakov soliton interactions ${ }^{10,27-29}$ were suggested as well. The concept underlying many of these methods is that a binary logical value can be associated with one of the soliton properties (such as position, timing, or frequency). An interaction with an additional soliton may alter these properties and change the binary value of the soliton bit.

A practical soliton switch or logic gate should be independent of the solitons' phases and polarization state and should allow cascading of several gate stages. In addition, it would be favorable if the participating bits (solitons) could be reused for further calculations; i.e., the logical operation should not modify the properties of the input solitons or annihilate them, which is one of the necessary conditions for a reversible logic.
The ability to cascade soliton gates and switches dictates a similarity between output and input bits (i.e., solitons with similar amplitude, width, frequency, etc.). Some of the previously suggested concepts such as trapping and dragging soliton gates required that the participating solitons be nonidentical to achieve the switching effect. In concepts based on four-wave mixing or second-harmonic generation the input soliton bits were destroyed in the course of generating the output soliton bit.

Although optical solitons do interact in Kerr media, utilizing this interaction to change the solitons' characteristics in a controlled manner is not simple. This interaction is elastic in the sense that far from the interaction region there is only a small shift in the solitons' position or phase (for spatial or temporal solitons). On the other hand, in the interaction region, the solitons' behavior is extremely dependent on their initial phase difference and polarization state. ${ }^{30-32}$ As a result, logic gates based on bulk soliton interactions require careful design and are generally sensitive to the solitons' initial state.

A favorable way to eliminate both polarization and phase dependence of the interactions between solitons is to use incoherent interactions between the solitons. Incoherent interactions occur, for example, if the variation of the phase difference is much faster than the response of the medium to electrical field (see Refs. 32 and 33 and references therein). Another possible realization of incoherent interaction is the employment of mutually incoherent solitons. Although incoherent soliton interactions are phase and polarization independent (in a nonbirefringent medium), much like coherent interaction they are elastic in a homogeneous Kerr medium (the Manakov system ${ }^{34}$ ) and are therefore difficult to exploit for logic operations and switching.

In this paper we study the incoherent interactions of solitons in an inhomogeneous Kerr medium comprising layers of media with different refractive indices. The 
study was based both on equations of motion describing the soliton particlelike trajectories in the inhomogeneous medium as well as by solving the nonlinear wave equation numerically. All-optical soliton switching and logic operations were demonstrated and exhibited efficient switching and low dissipation (to dispersive waves). All results were also validated by use of the beam propagation calculations.

In Section 2 we describe the framework of solitonsoliton and soliton-interface interactions by using a particle model. In Section 3 we construct solitonic logic elements by using the design rules derived in Section 2. In Section 4 we study the effect of loss on the performances and cascadability of the structures, and in Section 5 we conclude.

\section{FIELD EQUATION AND A PARTICLELIKE MODEL}

We analyze the propagation of several one-dimensional spatial solitons, which are interacting incoherently, in a Kerr medium that includes nonlinear interfaces (see Fig. 1). A nonlinear interface is formed in the boundary between two different Kerr media, each having, in general, different linear and nonlinear refractive indices.

In the framework of the paraxial approximation, the evolution of the electrical field envelope can be described by the nonlinear Schrödinger (NLS) equation:

$$
\begin{aligned}
& 2 i \beta k_{0} \frac{\partial E(z, x)}{\partial z}+\frac{\partial^{2} E(z, x)}{\partial x^{2}}-k_{0}^{2}\left\{\beta^{2}-\left[n_{0}^{2}(x)+2 n_{0}(x) n_{2}(x)\right.\right. \\
& \left.\left.\times|E(z, x)|^{2}\right]\right\} E(z, x)=0,
\end{aligned}
$$

where $E(z, x)$ is the electrical field, $k_{0}$ is the wave number in vacuum, $x$ is the transverse coordinate, $n_{0}(x)$ and $n_{2}(x)$ are the linear and nonlinear refractive indices, and $\beta$ is the $z$ propagation coefficient. The indices $n_{0}(x)$ and $n_{2}(x)$ depend on the layer structure; it is assumed that in each layer the indices are constant and are given by $n_{0 j}$ and $n_{2 j}$, where $j$ is the layer index (see Fig. 1).

Introducing the normalized variables $x^{\prime}=k_{0} x, z^{\prime}$ $=k_{0} z / 2 \beta$ and the scaled amplitude $E(x, z)$ $=\left(1 / n_{01} n_{21}\right)^{1 / 2} A(x, z) \exp \left[-i\left(\beta^{2}-n_{01}^{2}\right) z / 2 \beta\right]$ leads to the following normalized NLS equation (dropping the primes):

$$
\begin{gathered}
i \frac{\partial A}{\partial z}+\frac{\partial^{2} A}{\partial x^{2}}+2 A|A|^{2}=W A ; \\
W=\Delta_{j}-2\left(\alpha_{j}-1\right) \cdot|A|^{2}, \quad x \in L_{j},
\end{gathered}
$$

where $L_{j}$ represents the $j$ th layer in the structure (see Fig. $1), \Delta_{j}=n_{01}^{2}-n_{0 j}^{2}$, and $\alpha_{j}=n_{0 j} n_{2 j} /\left(n_{01} n_{21}\right)$. Equation (2) describes the propagation of the electrical field in a multilayered nonlinear medium. The left-hand side of Eq. (2) is the conventional NLS equation, while $W$ is an additional nonlinear potential generated by the layered structure. For $W=0$, i.e., when the nonlinear medium is homogeneous, Eq. (2) has an exact soliton solution:

$$
A(x, z)=A_{0} \sec h\left\{A_{0}[x-\bar{x}(z)]\right\} \exp [i v x / 2+\varphi(z)],
$$

where $A_{0}$ and $\bar{x}$ are the soliton amplitude and the average position, $v=\partial \bar{x} / \partial z$ is the soliton velocity, and $\varphi$ is the phase

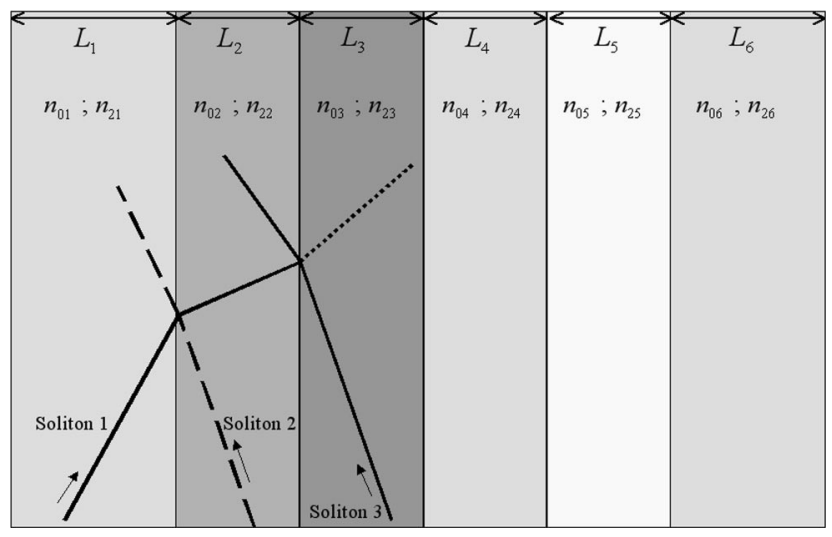

Fig. 1. Soliton interactions in a layered Kerr medium.

of the soliton satisfying $\partial \varphi / \partial z=A_{0}^{2}-v^{2} / 4$.

For arbitrary initial conditions, $A(x, z=0)$, Eq. (2) can be solved only numerically. However, if the initial conditions consist of well-separated solitons, the equation can be analyzed in the framework of soliton perturbation theory. In the perturbative approach adopted here, the solitons are treated as independent particles interacting with the interfaces and with each other. Combining the interaction (soliton-soliton and soliton-interface) establishes a complete particlelike model of the system.

Assuming the electrical field is a combination of $n$ mutually incoherent spatial solitons, a set of coupled NLS equations can be written to describe the evolution of the field in the multilayered nonlinear medium:

$$
\begin{gathered}
i \frac{\partial A_{m}}{\partial z}+\frac{\partial^{2} A_{m}}{\partial x^{2}}+2 A_{m}\left|A_{m}\right|^{2}=\left(\begin{array}{c}
W-2 \sum_{\substack{k=1 \ldots n \\
k \neq m}}\left|A_{k}\right|^{2}
\end{array}\right) A_{m}, \\
m=1 \ldots n,
\end{gathered}
$$

where $m$ is the soliton index and $W$ is defined in Eq. (2).

If the solitons are well separated and $\Delta_{j},\left(\alpha_{j}-1\right)$ are small, we can apply soliton perturbation theory to Eq. (4). In the framework of this theory, the solitons maintain the field profile (3), but the soliton parameters evolve during the propagation. The right-hand side of Eq. (4) can be treated as a perturbation source to the nonlinear Schrödinger equation [the left-hand side of Eq. (4)]. The perturbative approach yields four evolution equations for the soliton parameters $A_{0}, v, \bar{x}$, and $\varphi \cdot{ }^{35,36}$ While the equation for $A_{0}$ gives $A_{0}=$ constant, the equations for $\bar{x}$ and $v$ can be combined to yield an equation of motion for the soliton position:

$$
\frac{\mathrm{d}^{2} \bar{x}_{m}}{\mathrm{~d} z^{2}}=-2 p_{m}^{-1} \int_{-\infty}^{\infty} \frac{\partial R_{m}}{\partial x}\left|A_{m}\right|^{2} \mathrm{~d} x,
$$

where $\bar{x}_{m}, A_{m}$, and $p_{m}=\int_{-\infty}^{\infty}\left|A_{m}\right|^{2} \mathrm{~d} x$ are, respevctively, the position, field profile, and power of the $m$ th soliton. $R_{m}$ is the perturbation for the $m$ th soliton, which is given by the right-hand term of Eq. (4).

The force acting on each soliton particle is the result of two types of interaction, incoherent interactions with other solitons and interactions with the interfaces. The interaction between a soliton and an interface was analyzed by Aceves et al., ${ }^{37,38}$ who developed a one- 
dimensional particlelike model, resulting in an equation of motion for the average location of the soliton. The interface served as an induced effective potential barrier, which the particle (the soliton) may pass or be reflected from, according to its kinetic energy. The height of the potential barrier is directly proportional to the intensity of the soliton, and the kinetic energy depends on the socalled soliton velocity, which is a function of the incident angle.

Aceves model was employed to derive the force acting on a soliton propagating in a multilayered Kerr medium (see Fig. 1) simply by substituting the perturbation term $W$ defined in Eq. (2) into Eq. (5) instead of $R$. Incoherent interactions between solitons in a Kerr medium were analyzed previously by several groups. ${ }^{12,32,39-41}$ Unlike coherent interaction, solitons that interact incoherently in selffocusing media can only attract each other. The incoherent attraction between solitons of equal amplitude is given by ${ }^{12}$

$$
\begin{aligned}
f= & \frac{8 A_{0}^{3}}{\sinh ^{2}\left(A_{0} \Delta x\right)}\left\{A_{0} \Delta x\left[2 \operatorname{coth}^{2}\left(A_{0} \Delta x\right)+\sinh ^{-2}\left(A_{0} \Delta x\right)\right]\right. \\
& \left.-3 \operatorname{coth}\left(A_{0} \cdot \Delta x\right)\right\},
\end{aligned}
$$

where $A_{0}$ is the solitons' amplitude and $\Delta x$ is the separation between them. Using Eqs. (4), (5), and (6), we can derive a set of equations of motion for the soliton particles:

$$
\frac{\mathrm{d}^{2} \bar{x}_{m}}{\mathrm{~d} z^{2}}=-2 p_{m}^{-1} \int_{-\infty}^{\infty} \frac{\partial W}{\partial x}\left|A_{m}\right|^{2} \mathrm{~d} x+\sum_{\substack{k=1 \ldots n \\ k \neq m}} f_{m k},
$$

where $f_{m k}$ is the force acting on soliton $m$, caused by the presence of soliton $k$, according to Eq. (6).

\section{CONSTRUCTION OF SOLITON-BASED LOGIC GATES AND SWITCHES}

The incoherent interactions between solitons within a homogeneous medium do not mediate any information transfer between the solitons (except for a small phase
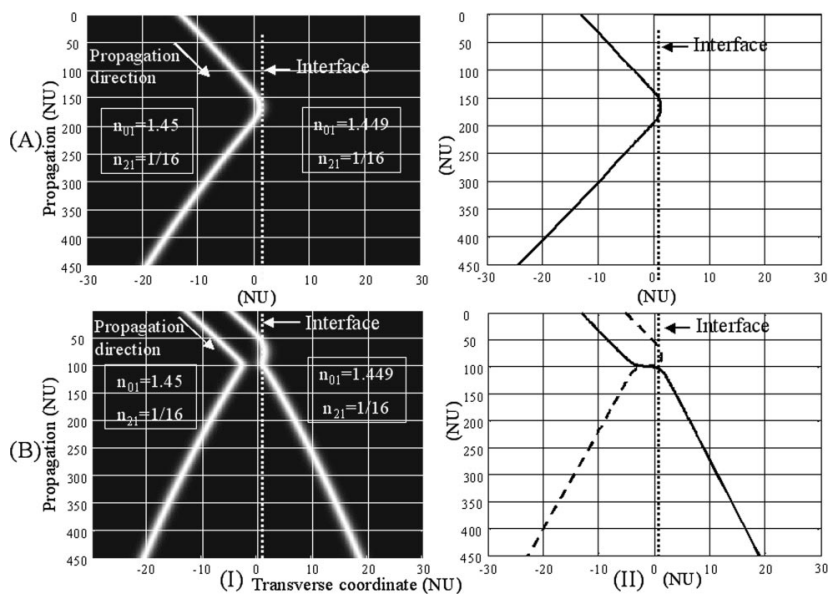

Fig. 2. All-optical soliton switch. (A) Control soliton turned off; the signal soliton is reflected from the interface. (B) Control soliton turned on; the signal soliton is transmitted through the interface. column (I), BPM simulation; (II), particlelike model. $n_{01}$ $=1.45, n_{02}=1.449, n_{21}=1 / 16, n_{22}=1 / 16$, soliton velocity 0.1 .
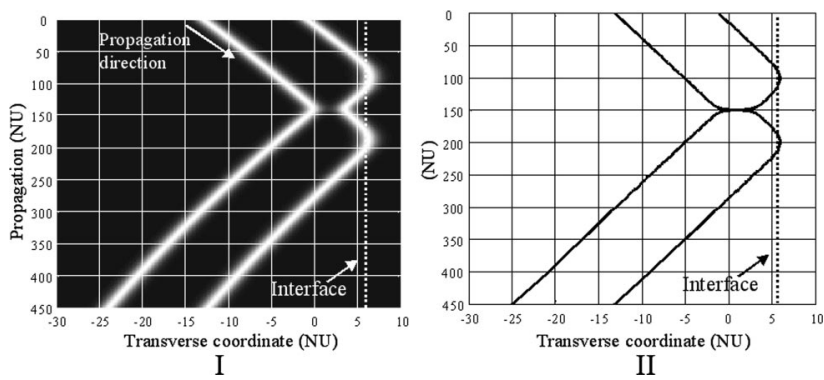

Fig. 3. Two incoherent solitons interact with an interface with large initial separation: (I) BPM, (II) particlelike model. The structure and soliton parameters are the same as in Fig. 2.

shift); i.e., the interactions are elastic. ${ }^{39-41}$ If, however, the interaction takes place in the vicinity of a nonlinear interface, it may significantly change the trajectories of the solitons. ${ }^{12}$ For example, the behavior of a soliton impinging at an oblique angle on an interface can be toggled from reflection to transmission by turning an additional soliton on and off. Figure 2 depicts an all-optical soliton switch that is based on a soliton interaction near an interface. The switch employs two solitons, a signal soliton and a control soliton. When the control soliton is turned off [Fig. 2(a)], the signal soliton is reflected from the interface. However, when the control soliton is turned on [Fig. 2(b)], the interface becomes transparent for the signal soliton, which traverses the interface and maintains its propagation in the other medium while maintaining its shape and identity. This effect is the result of lowering the effective potential barrier encountered by the signal soliton assisted by the control soliton, ${ }^{12}$ which makes its transmission possible. It is important to observe that the particlelike analysis gave similar results to the propagation calculations (Fig. 2), although the model probably fails locally within the close interaction region. The amplitudes of the solitons after switching [Fig. 2(b)] were found to be identical (within the limits of the numerical calculation) to their amplitudes at $z=0$, indicating negligible radiation losses.

Note that the propagation coordinate $(z)$ and the transverse coordinate $(x)$ are not normalized in the same manner. As a result, some of the interactions shown in Fig. 2 and in Figs. 3-12 below might appear, incorrectly, to occur in the nonparaxial limit. The real incidence angles of the solitons in these figures are in the range $3^{\circ}-7^{\circ}$, which is well within the paraxial approximation regime.

Figure 3 depicts the solitons' trajectories (with same amplitudes and velocities as in Fig. 2) when the interaction takes place far from the interface, showing that for bulk interactions the trajectories are hardly affected by the presence of the other soliton.

These two types of soliton interaction (interface assisted and bulk) are the building blocks of an all-optical processing device. Soliton bits can affect each other if they interact near the interface or avoid each other if the interaction takes place in a homogeneous medium. The following logic elements' operation is based on controlled modifications of the soliton trajectories. All the results were obtained by employing the particlelike model, and all were verified by the beam propagation method. 


\section{A. Soliton AND-OR Gates}

The scheme is illustrated in Fig. 4. Two solitons, which serve as the input bits (soliton "1," no soliton "0") of the gate, are incident at an oblique angle on both sides of a nonlinear interface. The output of the device (marked by AND-OR operation at the bottom of Fig. 4) is considered "1" if one of the solitons reaches the output port and "0" otherwise. The solitons' interactions at the interface would form an AND-OR operation if the equivalent potential [according to Eq. (7)] of one of the interface sides were higher than the potential of the other side. Such an equivalent potential can be engineered, for example, if the linear refractive index of the medium on the left-hand side is higher than that of the medium on the right-hand side of the interface while their nonlinear refractive indices are equal. The difference must be small enough to ensure that the solitons on both sides of the interface are similar enough to keep radiation losses as small as possible. The potential barrier induced by the interface in this case is given by Eq. $(8)^{37}$ :

$$
\Delta U=\frac{2}{3} A_{0}^{2} \frac{(1-\alpha)^{2}}{\alpha}, \quad \text { where } \quad \alpha=\frac{n_{01} n_{21}}{n_{02} n_{22}} .
$$

The suggested gate operates as follows: The incident angle of the soliton on the left-hand side of the interface (soliton A in Fig. 4) is below the critical angle; i.e., if only this soliton impinges on the interface, it will be reflected. Soliton B is experiencing the higher side of the potential barrier induced by the interface (the right-hand side), and therefore will always pass to the lower-level side of the potential (the left-hand side) regardless of its incidence angle. If, however, both solitons coexist, soliton A would also cross the barrier to the right-hand side, while soliton B would pass to the left.

Figure 5(a) depicts Beam Propagation Method (BPM) simulations and, Fig. 5(b) shows the results of the particlelike model for three input pairs as described in the previous paragraph (when both input bits are " 0 ," the output is obviously "0"). The parameters of the structure are defined in the figure caption. It can be seen that the outcome in which a soliton propagates on the right-hand side of the interface following the interaction occurs only when

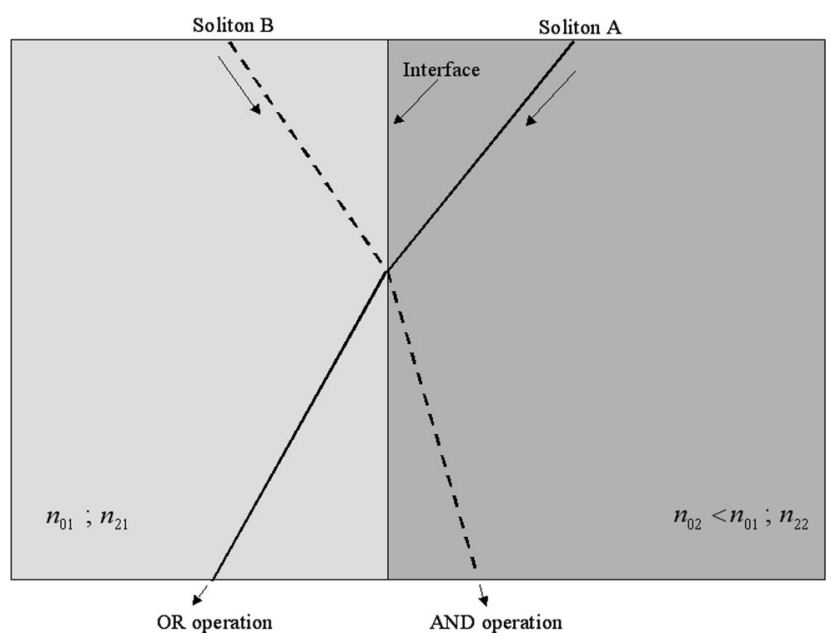

Fig. 4. All-optical soliton AND-OR gate.
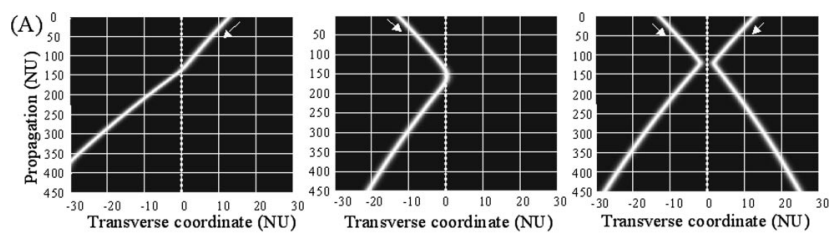

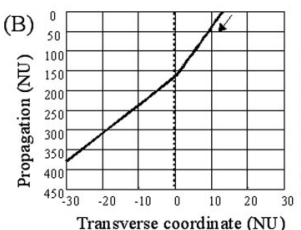

(I)

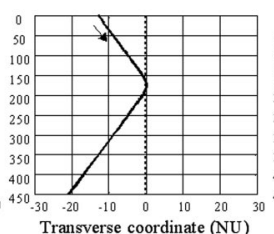

(II)

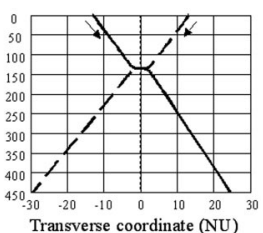

(III)
Fig. 5. Operation of AND-OR gate: (I) $A=1, B=0$; (II) $A=0, B=1$; (III) $A=1, B=1$. (A) BPM simulations, (B) Particlelike model. $n_{01}=1.45, n_{02}=1.449, n_{21}=1 / 16, n_{22}=1 / 16$.

both solitons are present. The existence or non-existence of a soliton on the right-hand side of the interface is a result of an AND operation between the input solitons. In a similar fashion, a soliton will always propagate after the interaction on the left-hand side of the interface unless both bits are " 0 ," thus corresponding to an oR logical operation. The same structure serves as an AND and an OR gate simultaneously. As for the soliton switch, no apparent radiation losses were generated by the interaction.

The results of the particlelike design calculations (row $B$ in Fig. 5) exhibited a good agreement with the verification results of the BPM simulations. Thus the particlelike model provides a fast and simple tool for designing interface-based soliton switches and logic gates.

\section{B. All-Optical Not Gate}

An inverter (NOT gate) is an essential building block for a complete set of logic functionality. In order to realize a NOT operation, a gate soliton is required to probe the input soliton. Although this operation is apparently performed by the soliton switch presented in the beginning of this section, the outcome is not adequate. Without the signal the control soliton is reflected from the interface, generating a " 1 ." When the signal soliton is inserted, the latter passes the interface, but the control is still reflected, again generating a "1" (see Fig. 2).

A simple realization of a solitonic inverter consists in a similar layer structure described for the AND-OR gate, however, with different linear and nonlinear refractive indices to make a trapped surface soliton possible ${ }^{37}$ Figure 6 depicts the induced potential of an interface as defined by $n_{01}=1.5, n_{21}=0.0455, n_{02}=1.45, n_{22}=0.06$. Here there is a local minimum in the induced potential, permitting the existence of an interface-trapped soliton. The structure is realizing a NOT operation in the following way: The trapped soliton serves as the gate soliton. If a signal soliton hits the interface at an oblique angle, it pulls the gate soliton out of the potential well. Thus, the existence of the gate soliton at the interface is monitored as the output of the inverter. Figure 7 depicts a BPM simulation of the device when the signal soliton exists (" 1 " input). The signalsoliton pulls the gate soliton out of the interface trap, and both solitons continue to propagate away from the interface, yielding a "0" output. Without the signal soliton, the gate soliton would continue propagating along the inter- 


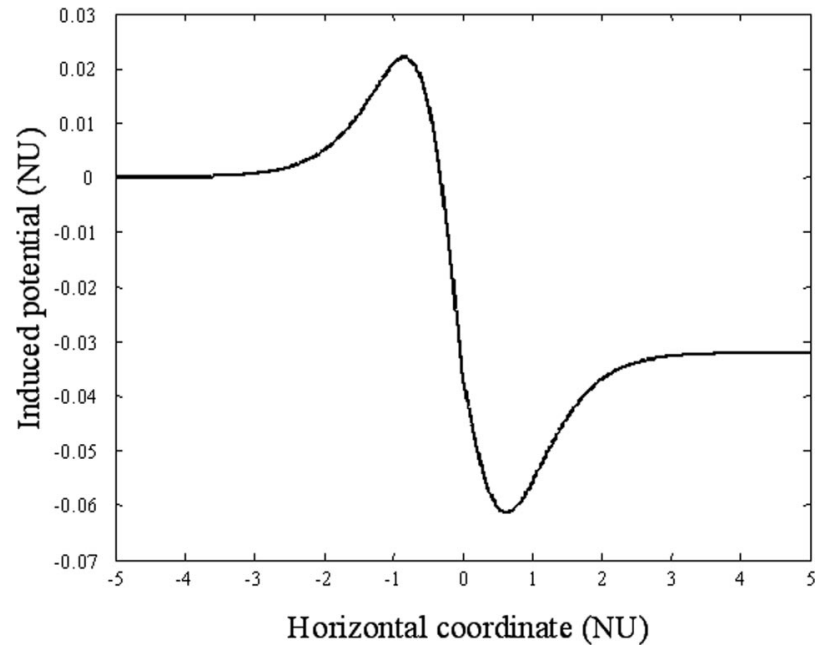

Fig. 6. Induced potential of an interface defined by $n_{01}=1.5$, $n_{21}=0.0455, n_{02}=1.45, n_{22}=0.06$.

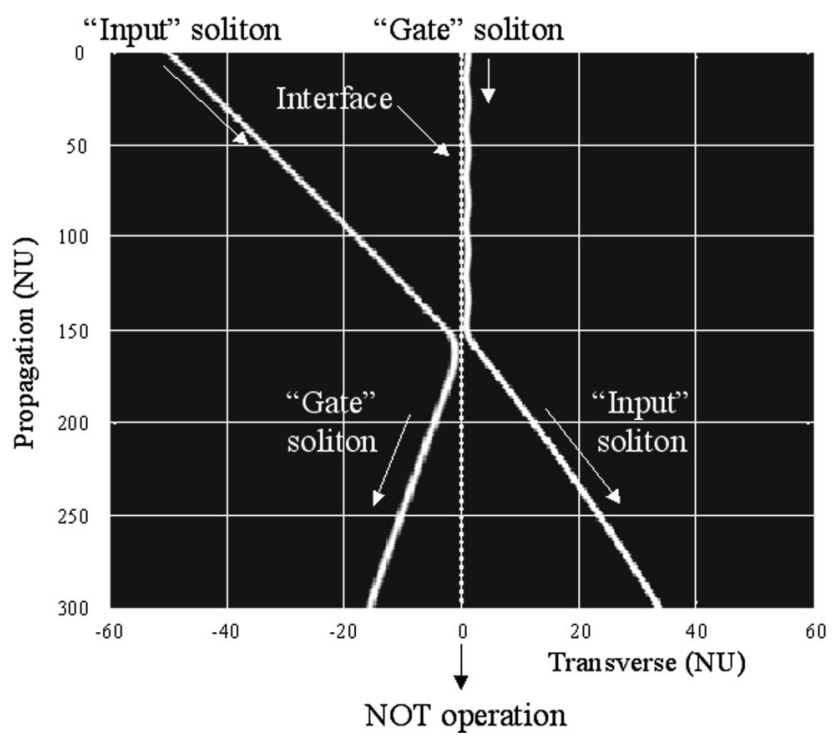

Fig. 7. BPM simulation of a single-interface soliton inverter for "1" input.

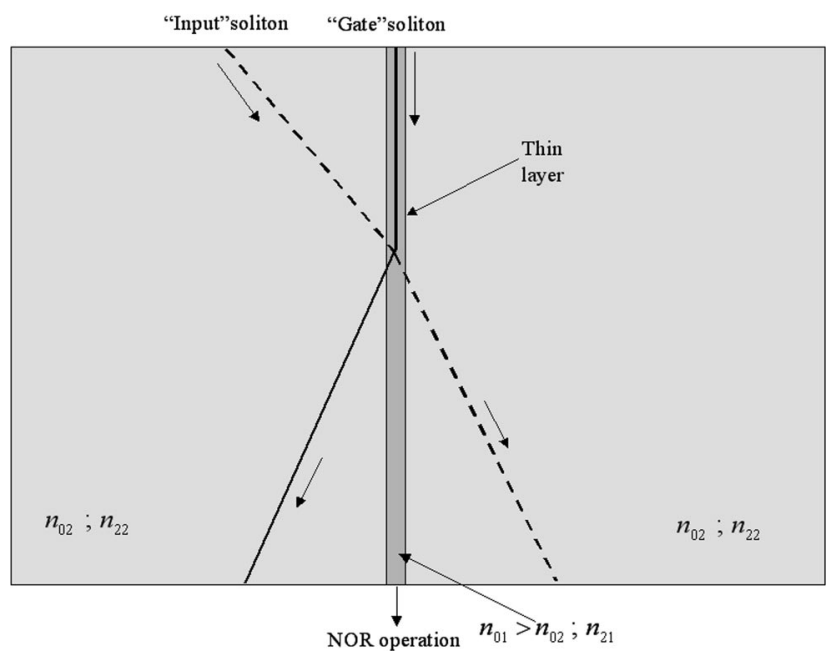

Fig. 8. Double-interface NOT gate. face, yielding a "1" output. It should be emphasized that despite the relatively large index contrast the calculated radiation losses induced by the interactions were 2 $\times 10^{-3} \mathrm{~dB}$.

An enhanced configuration is to use a double-interface structure constructed by a thin layer of higher linear index surrounded by medium with lower linear index, both with a similar nonlinear refractive index (Fig. 8). Unlike the single-interface scheme, where the existence and position of the potential minimum are highly sensitive to the indices, the double interface ensures the existence of the potential minimum and fixes its position. The interaction of a single soliton with the double-interface structure was studied by Kivshar and Quiroga-Teixeiro, ${ }^{42}$ who found that there is a single minimum of the induced potential if the following conditions are satisfied:

$$
\left(1-\frac{n_{0 \text { in }} n_{2 \text { in }}}{n_{\text {0out }} n_{\text {2out }}}\right)\left(n_{\text {0in }}^{2}-n_{\text {out }}^{2}\right)<0, \quad\left(n_{\text {0in }}^{2}-n_{\text {out }}^{2}\right)>0,
$$

where $n_{j \text { in }}$ and $n_{j o u t}$ are the linear $(j=0)$ and nonlinear $(j=2)$ refractive indices of the thin layer and the bulk, respectively.

Figure 9 shows both a BPM simulation and the results of a particlelike model for the double-interface NOT gate. The simulation parameters are defined in the figure caption. The results of the particlelike model qualitatively agree with the BPM results; the differences probably originate in the inability of the force, Eq. (5), to describe the interaction between the solitons accurately at small separation distances (see, for example, Ref. 43).

For a given set of media, the NOT gate structure can be optimized by changing the input soliton velocity (incident angle) and the layer width. To make the device as short as possible, maximizing the transverse velocity of the gate soliton is beneficial. If the layer is very thin, its induced potential is negligible, and the interaction between the solitons is similar to the bulk interaction (zero gate soliton velocity). If, however, the layer is very thick, the interaction between the solitons would practically take place in a homogeneous medium (the layer), and the gate soliton would remain trapped inside the potential well. An upper limit on the layer width can be estimated according to the position shift that is introduced by a soliton interaction in the homogeneous medium of the layer (similar to the phase shift in temporal soliton interactions). If half of the layer width is larger than this shift, the gate soliton would remain trapped in the layer. The
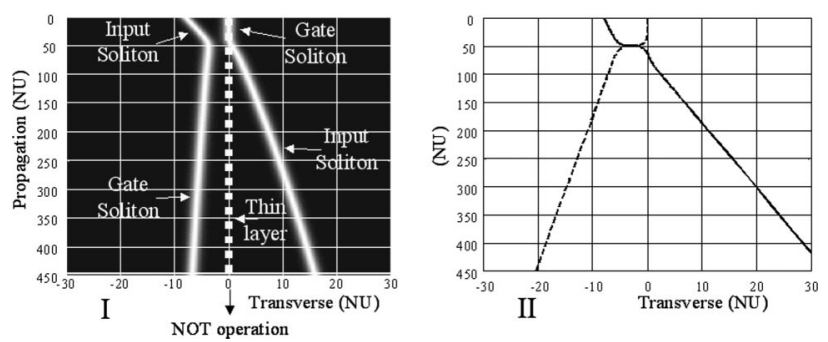

Fig. 9. Double-interface inverter with "1" input. (I) BPM simulations. (II) Particlelike model. The structure and soliton parameters are the same as in Fig. 5. 


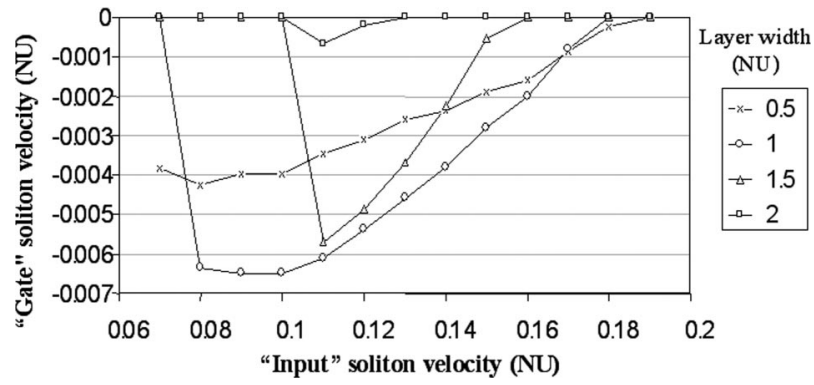

Fig. 10. Gate soliton velocity as a function of the input soliton velocity for various layer widths.

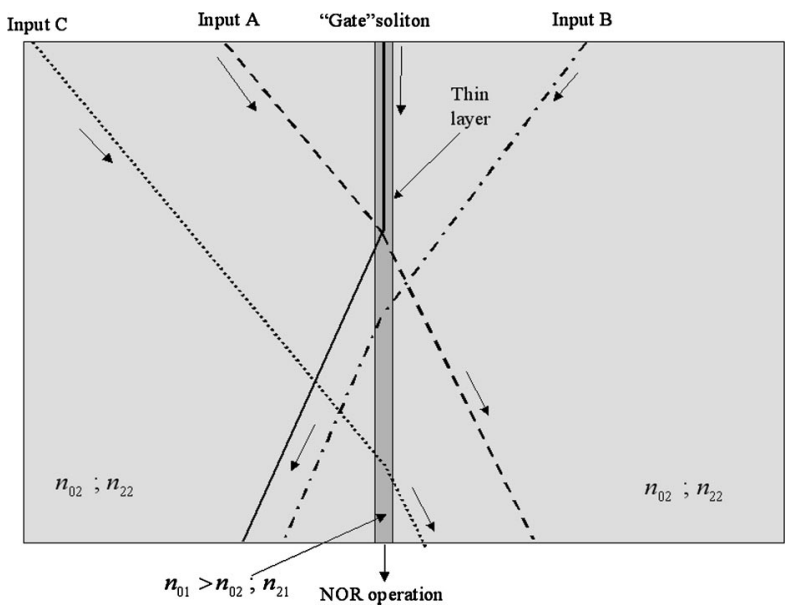

Fig. 11. Schematic of an all-optical soliton NOR gate.

position shift of the solitons is given by ${ }^{10}$

$$
\Delta=-\ln (v / 4) .
$$

Therefore there is an optimal layer width for which the gate soliton velocity, following the interaction, is maximal. Similarly, there is an optimal value for the input soliton velocity. A slow (shallow incident angle) soliton has low kinetic energy and would not be able to extract the gate soliton from within the potential well. The depth of the potential well is given by its value at the middle of the layer ${ }^{42}$ :

$$
\begin{aligned}
U_{\min }= & {\left[\Delta+A_{0}^{2}(1-\alpha)\right] \tanh \left(\frac{A_{0} L}{2}\right) } \\
& -\frac{2}{3} A_{0}^{2} \cdot(1-\alpha) \tanh ^{3}\left(\frac{A_{0} L}{2}\right),
\end{aligned}
$$

where $L$ is the layer thickness, $\Delta_{j}=n_{02}^{2}-n_{01}^{2}$, and $\alpha$ $=\left(n_{01} n_{21}\right) /\left(n_{02} n_{22}\right)$ (see Fig. 8). This sets a lower limit on the velocity of the gate soliton $v$ [as defined in Eq. (3)], $v^{2} / 2>\left|U_{\min }\right|$. A fast input soliton would have a small interaction length with the gate soliton and would have a rather small effect on the gate soliton.

Figure 10 depicts the dependence of the gate soliton velocity on the input soliton velocity for various layer widths. Zero velocity indicates that the gate soliton remained trapped inside the layer after the interaction, although the soliton position may oscillate around the layer center. As expected, by increasing the input soliton velocity above a certain level ( $\sim 0.13$ for the specific structure), we decrease the gate soliton velocity because of the smaller interaction length. On the other hand, when the signal soliton is slow, the gate soliton indeed remains trapped inside the potential well except for in very thin layers $(d \leqslant 1)$ in which the induced potential well is shallow. For the examined structure, the optimal set of parameters is $v$ (input) $=0.1$ and $d=1$, which yields a gate soliton velocity of $\sim 6.5 \times 10^{-3}$.

Figure 10 also indicates that the proposed structure can operate successfully in a wide range of incidence angles and inner layer thicknesses. At telecom wavelengths $(\lambda \sim 1.55 \mu \mathrm{m})$ the optimal layer thickness (for $n_{01}=1.45$ ) is $0.5 \mu \mathrm{m}$, and the incidence angles (relative to the interface plane), for which the gate operates, range between $1.6^{\circ}$ and $3.2^{\circ}$. This is a reasonable operating range to achieve and does not require ultrafine tuning of the beam's incident angle.

\section{All-Optical NOR Gate}

One of the main advantages of the NOT gate structure described in Subsection 3.B is that it can be easily expanded to a multiple-port NOR gate. Figure 11 depicts a schematic of the suggested NOR gate. A NOR gate output is "0" if one (or more) of its input signals is "1." The suggested structure (Fig. 11) implements the NOR functionality in the following manner: If one of the input solitons exists (i.e., " 1 "), the gate soliton would be pulled out of the potential well and the gate output would be " 0 ." Because of the stepped structure of the soliton inputs, the existence of other input solitons would not affect the result, since they would interact with the gate soliton in the bulk and would not change its velocity. For the soliton NOR gate to function properly there is a limit on the minimal lateral distance between the input solitons' positions, which depends on the input solitons' incident angle, amplitude, etc.

Figure 12 depicts a BPM simulation of a triple-input NOR gate, where two input signals $(\mathrm{A}, \mathrm{C})$ are " 1 " and the third (B) is "0." It can be easily seen that the rightmost

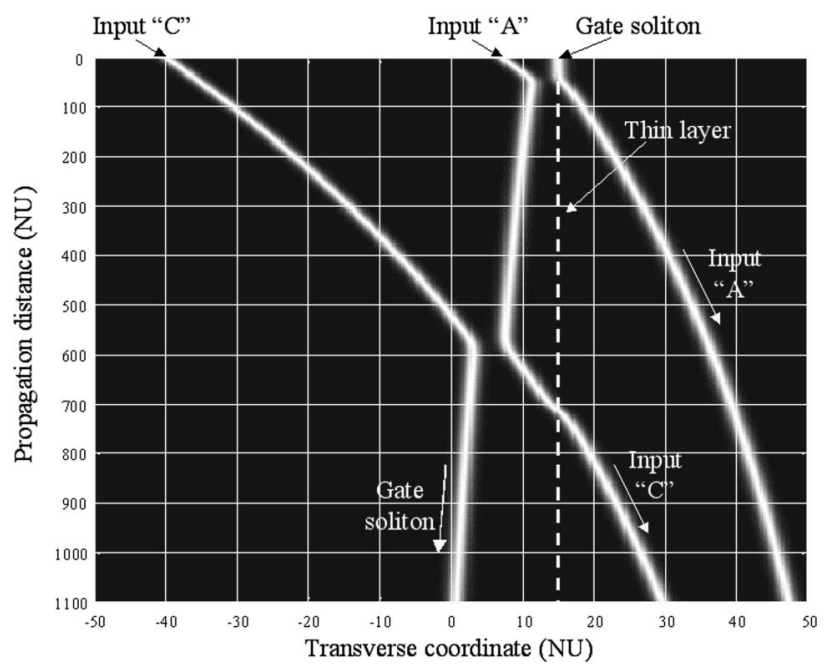

Fig. 12. BPM simulation of a three-input soliton NOR gate. Media parameters are as in Fig. 5. 
soliton extracts the gate soliton from the middle layer while the other soliton interacts with the gate soliton in a (practically) homogeneous medium and therefore does not change its velocity.

\section{EFFECT OF LOSS MECHANISMS}

The switches and logic gates presented here are based on the nonlinear interaction between solitons and between a soliton and an interface. The outcome of these interactions is, therefore, sensitive to the amplitudes of the solitons. While the radiation losses due to the interaction of the solitons with the interfaces are negligible, scattering losses and material absorption generate continuous attenuation of the solitons' amplitude. The reduced amplitude alters the dynamics of both the soliton-soliton and the soliton-interface interactions and might impair the operation of the logic gate. Practically, the losses limit the overall distance each soliton can propagate without losing its effectiveness or, in other words, the losses limit the cascadability of the soliton gates.

The number of gates that can be cascaded (without optical amplification) depends on the losses, the length of each gate, and the minimal soliton amplitude required for the logic operation. The length of logic gate presented here varied between 300 and 500 normalized units (NU), which corresponds approximately to $250-400 \mu \mathrm{m}$ at telecom wavelengths. By simulating the all-optical gates for various soliton amplitudes, we found that the basic structures presented here, even without additional optimization, operate properly if the amplitudes are between 0.8 and 1 (NU). For a reasonable propagation loss of $\sim 2 \mathrm{~dB} / \mathrm{cm}$, this range enables us to cascade at least 20 gates.

The loss problem can be partially overcome, for example, by modifying the design of each gate to account for the reduced intensity or by introducing optical gain to the device. Nevertheless, both concepts incorporate inherent limitations. Modifying each gate requires exact tailoring of the material structure for each desired gate combination. Optical gain introduces additional noise into the system and generates jitter in the solitons' positions and velocities, which in turn limits the number of gates that could be cascaded. The analysis of the effect of this jitter is, however, beyond the scope of this paper.

\section{DISCUSSION AND SUMMARY}

We studied the incoherent interactions of spatial solitons in a multilayered Kerr medium comprising layers of different Kerr media. We showed that soliton interactions in the vicinity of nonlinear interfaces could be used to achieve all-optical switching and computing. Architecture for reversible AND, OR, NOT, and NOR gates was presented and analyzed. The dynamics of the system can be qualitatively (and in some cases even accurately) described by a particlelike model based on soliton perturbation theory.

The key element was the inhomogeneity of the nonlinear medium (the existence of the interfaces), which enabled permanent momentum transfer between the solitons. Although the different media support different solitons, the difference in the media parameters was de- signed to be small, and thus the solitons are similar enough to allow low-loss intermedia transmission. The exchange of momentum boosted one of the solitons to change its trajectory with respect to the interface (crossing versus reflection, etc.) and thus to exhibit switching or a logic operation. The interacting solitons maintained their soliton identity and shape after the collision and could therefore be reused for the next stage of soliton gates. The output solitons of the gates presented here are identical to the input solitons; the gates could be easily cascaded to achieve complex operations.

The effect of scattering and absorption losses on the performance and the cascadability of the gates was analyzed. Even without optimization it is possible to cascade at least 20 devices without their performances deteriorating. More devices can be cascaded by introducing optical gain.

Experiments to demonstrate this behavior can be conducted with slab waveguides fabricated in GaAs with below-midgap nonlinearity (or with low-growth temperature GaAs) or with dielectric highly nonlinear media, such as a liquid crystal or nonlinear organic material. For these media we showed that at peak amplitudes of $0.8 \mathrm{~V} / \mathrm{mm}$ and with a typical nonlinear coefficient $n_{2}$ of $\sim 10^{-9} \mathrm{~m}^{2} / \mathrm{W}$, using power levels of $\sim 2 \mathrm{~kW} / \mathrm{cm}^{2}$ is enough to induce the required phenomena, as can also be seen from Fig. 2 of Ref. 12, which illustrates the barrier repulsion effects at these conditions.

Jacob Scheuer may be reached at koby@caltech.edu; Meir Orenstein may be reached at meiro@ee.technion.ac.il.

1. T. K. Gustafson and P. W. Smith, eds., Photonic Switching (Springer-Verlag, Berlin, 1988).

2. N. J. Doran and D. Wood, "Soliton processing element for all-optical switching and logic," J. Opt. Soc. Am. B 4, 18431846 (1987).

3. T. T. Shi and S. Chi, "Nonlinear photonic switching by using the spatial soliton collision," Opt. Lett. 15, 1123-1125 (1990).

4. M. N. Islam, C. E. Soccolich, C. J. Chen, K. S. Kim, J. R. Simpson, and U. C. Paek, "All-optical inverter with one picojoule switching energy," Electron. Lett. 27, 130-132 (1991).

5. M. N. Islam and C. E. Soccolich, "Billiard-ball soliton interaction gates," Opt. Lett. 16, 1490-1492 (1991).

6. S. R. Friberg, "Demonstration of colliding-soliton all-optical switching," Appl. Phys. Lett. 63, 429-431 (1993).

7. G. R. Williams, M. Vaziri, K. H. Ahn, B. C. Barnett, and M N. Islam, "Soliton logic gate using low-birefringence fiber in a nonlinear loop mirror," Opt. Lett. 20, 1671-1673 (1995).

8. Y. Oh, J. W. Haus, and R. L. Fork, "Soliton-repulsion logic gate," Opt. Lett. 21, 315-317 (1996).

9. K. H. Ahn, M. Vaziri, B. C. Barnett, G. R. Williams, X. D. Cao, M. N. Islam, B. Malo, K. O. Hill, and D. Q. Chowdhury, "Experimental demonstration of low-latency fiber soliton logic gate,” J. Lightwave Technol. 14, 1768-1775 (1996).

10. R. Radhakrishman, M. Lakshmanan, and J. Hietarinta, "Inelastic collision and switching of coupled bright solitons in optical fibers," Phys. Rev. E 56, 2213-2216 (1997).

11. L. Lefort and A. Barthelemy, "All-optical demultiplexing of a signal using collision and waveguiding of spatial solitons," IEEE Photonics Technol. Lett. 9, 1364-1366 (1997).

12. J. Scheuer and M. Orenstein, "Interactions and switching of spatial soliton pairs in the vicinity of a nonlinear interface," Opt. Lett. 24, 1735-1737 (1999).

13. O. V. Kolokoltsev, R. Salas, and V. Vountesmeri, "All-optical 
phase-independent logic elements based on phase shift induced by coherent soliton collisions," J. Lightwave Technol. 20, 1048-1053 (2002).

14. M. Peccianti, C. Conti, G. Assanto, A. De Luca, and G. Umeton, "All-optical switching and logic gating with spatial solitons in liquid crystal," Appl. Phys. Lett. 81, 3335-3337 (2002).

15. G. R. Collecutt and P. D. Drummond, "Digital response with femtosecond resolution in an optical AND gate," Opt. Commun. 184, 237-243 (2000).

16. X. Liu, K. Beckwitt, and F. Wise, "Noncollinear generation of optical spatiotemporal solitons and application to ultrafast digital logic," Phys. Rev. E 61, R4772-R4725 (2000).

17. M. N. Islam, "All-optical cascadable NOR gate with gain," Opt. Lett. 15, 417-419 (1990).

18. Q. Wang, P. K. A. Wai, C. J. Chen, and C. R. Menyuk, "Numerical modeling of soliton dragging logic gates," J. Opt. Soc. Am. B 10, 2030-2039 (1993).

19. R. McLeod, K. Wagner, and S. Blair, " $(3+1)$-dimensional optical soliton dragging logic," Phys. Rev. A 52, 3254-3278 (1995).

20. M. Bertolotti, A. D'Andrea, E. Fazio, M. Zitelli, A. Carrera, G. Chiaretti, and N. G. Sanvito, "Experimental observation of spatial soliton dragging in a planar glass waveguides," Opt. Commun. 168, 399-403 (1999).

21. S. Saxena, P. K. A. Wai, C. J. Chen, and C. R. Menyuk, "Modeling of soliton-dragging logic gates with gain," Opt. Lett. 19, 1370-1372 (1994).

22. S. Blair, K. Wagner, and R. McLeod, "Asymmetric spatial soliton dragging," Opt. Lett. 19, 1943-1945 (1994).

23. M. N. Islam, C. R. Menyuk, C. J. Chen, and C. E. Soccolich, "Chirp mechanisms in soliton-dragging logic gates," Opt. Lett. 18, 214-216 (1991).

24. C. E. Soccolich, M. W. Chbat, M. N. Islam, and P. R. Prucnal, "Cascade of ultrafast soliton-dragging and trapping logic gates," IEEE Photonics Technol. Lett. 4, 1043-1046 (1992).

25. M. W. Chbat, B. Hong, M. N. Islam, C. E. Soccolich, and P. R. Prucnal, "Ultrafast soliton-trapping AND gate," J. Lightwave Technol. 10, 2011-2016 (1992).

26. M. H. Jakubowski, K. Steiglitz, and R. Squier, "Information transfer between solitary waves in the saturable Schrödinger equation," Phys. Rev. E 56, 7267-7272 (1997).

27. M. H. Jakubowski, K. Steiglitz, and R. Squier, "State transformation of colliding optical soliton and possible application to computation in bulk media," Phys. Rev. E 58, 67526758 (1998).
28. K. Steiglitz, "Time-gated Manakov spatial solitons are computationally universal," Phys. Rev. E 63, 016608 (2000).

29. C. Anastassiou, M. Segev, K. Steiglitz, J. A. Giordmaine, M. Mitchell, M. Shih, S. Lan, and J. Martin, "Energy-exchange interactions between colliding vector solitons," Phys. Rev. Lett. 83, 2332-2335 (1999).

30. J. P. Gordon, "Interaction forces among solitons in optical fibers," Opt. Lett. 8, 596-598 (1983).

31. G. P. Agrawal, Nonlinear Fiber Optics (Academic, San Diego, 1989).

32. G. I. Stegman and M. Segev, "Optical spatial solitons and their interactions: universality and diversity," Science 286, 1518-1523 (1999).

33. M. Segev, "Optical spatial solitons," Opt. Quantum Electron. 30, 503-533 (1998).

34. S. V. Manakov, "On the theory of two-dimensional stationary self focusing of electromagnetic waves," Sov. Phys. JETP 38, 248-253 (1974).

35. V. I. Karpman and E. M. Maslov, "Perturbation theory for solitons," Sov. Phys. JETP 48, 281-291 (1977).

36. H. A. Haus and Y. Lai, "Quantum theory of soliton squeezing: a linearized approach,” J. Opt. Soc. Am. B 7, 386-392 (1990).

37. A. B. Aceves, J. V. Moloney, and A. C. Newell, "Theory of light-beam propagation at nonlinear interfaces. I. Equivalent-particle theory for a single interface," Phys. Rev. A 39, 1809-1827 (1989).

38. A. B. Aceves, J. V. Moloney, and A. C. Newell, "Theory of light-beam propagation at nonlinear interfaces. II. Multiple-particle and multiple-interface extensions," Phys. Rev. A 39, 1828-1840 (1989).

39. D. Anderson and M. Lisak, "Variational approach to incoherent two-soliton interaction," Phys. Scr. 33, 193-196 (1986).

40. M. Karlsson, D. Anderson, A. Höök, and M. Lisak, "A variational approach to optical soliton collisions," Phys. Scr. 50, 265-270 (1994).

41. A. Berntson, D. Anderson, and M. Lisak, "Analysis of coherent and incoherent interactions of amplitude shifted solitons in optical fibers," Phys. Scr. 52, 544-553 (1995).

42. Y. S. Kivshar and M. L. Quiroga-Teixeiro, "Light-beam propagation at planar thin-film nonlinear waveguides," Phys. Rev. A 48, 4750-4757 (1993).

43. D. De Angelis and S. Wabnitz, "Interactions of orthogonally polarized solitons in optical fibers," Opt. Commun. 125, 186-196 (1996). 